\title{
Tribologische Untersuchungen am Förderzahnriemen
}

\author{
Prof. DR.- ING. KLAUS NENDEL, \\ DIPL.- ING. HENDRIK KADEN \\ TU CHEMNITZ, FAKULTÄT FÜR MASCHINENBAU, PROFESSUR FÖRDERTECHNIK
}

\begin{abstract}
Zusammenfassung
Zahnriemenfördersysteme haben aufgrund ihrer wirtschaftlichen und technischen Vorteile beim Transport von Stückgütern ein breites Anwendungsfeld in den unterschiedlichen Bereichen der Industrie gefunden und gewinnen weiterhin an Bedeutung. Die Auslegung der Systeme beschränkt sich gegenwärtig im Wesentlichen auf die Zugstrang- und die Zahnfußfestigkeit des Zahnriemens. Grundlagen der Berechnungen sind oft recht vage Aussagen zur Höhe des Reibwertes zwischen dem Zahnriemen und dessen Stützschiene. Die Erhöhung der Kontakttemperatur durch die eingebrachte Reibleistung wird meist völlig vernachlässigt. In der Praxis wird oftmals auf Erfahrungswerte zurückgegriffen, wobei die Gefahr der Über- bzw. Unterdimensionierung mit erheblichen Auswirkungen auf die Lebensdauer, das Verschleißverhalten und die Betriebssicherheit besteht.
\end{abstract}

\begin{abstract}
Because of their economical and technical advantages in transporting piece goods timing belt conveying systems are used in a wide field of application in diverse areas of the industry and are still gaining in importance. Currently the dimensioning of the systems restrains primarily at the cord resistance and the resistance of the base of the cleat of the timing belt. Quite vague statements about the extent of the coefficient of friction between the timing belt and its support rail are used as basic principle of the calculation. Mostly the increase of the contact temperature through the inserted friction will be totally disregarded. The practice very often bases on experience. Thereby the danger of over-sizing and under-sizing, as the case may be, might have considerable consequences at the lifetime, the wear behaviour and the operating reliability.
\end{abstract}

\section{Anwendung von Zahnriemenförderern}

Das Einsatzgebiet der Zahnriemen ist neben der Antriebstechnik in zunehmendem Maße die Fördertechnik, wo diese als Zug- und Tragmittel für den Stückguttransport zur Anwendung kommen. Der Grund dieser Entwicklung lässt sich mit den günstigen Eigenschaften dieser Maschinenelemente erklären. Besonders zu erwähnen sind dabei der geräuscharme Lauf, die geringe Masse und die niedrigen Kosten in Anschaffung und Wartung.

Der synchrone Lauf, der mit Zahnriemen wie auch mit Förderketten realisierbar ist, ist ein weiterer wesentlicher Vorteil. Dabei übernehmen die robusten Förderketten den Bereich der Fördertechnik, in dem große Kräfte übertragen werden müssen und stark schmutzintensive Umgebungsbedingungen vorherrschen. Haupteinsatzgebiete der Zahnriemenförderer ist der Bereich der empfindlicheren Güter mit relativ geringen Massen, wobei sich immer mehr abzeichnet, dass auch Einsatzgebiete mit schweren Werkzeugträgern erschlossen werden.

Die Transportzahnriemen müssen bei dem Einsatz zahnseitig abgestützt werden, um die Gutmasse aufnehmen zu können. Stückgüter können von Zahnriemen durch Kraft- oder Formschluss transportiert werden. Der Einsatz ist von den technologischen Erfordernissen und der Art des Transportgutes abhängig.

Formschluss wird meist über aufgeschweißte Formelemente / Mitnehmer realisiert. Diese Art des Transportes wird verwendet, wenn Teile:

- $\quad$ vereinzelt,

- genau positioniert,

- zeitlich exakt getaktet,

- über starke Steigungen bis hin zum vertikalen transportiert werden müssen,

- $\quad$ bzw. sich gegenseitig nicht berühren dürfen. 


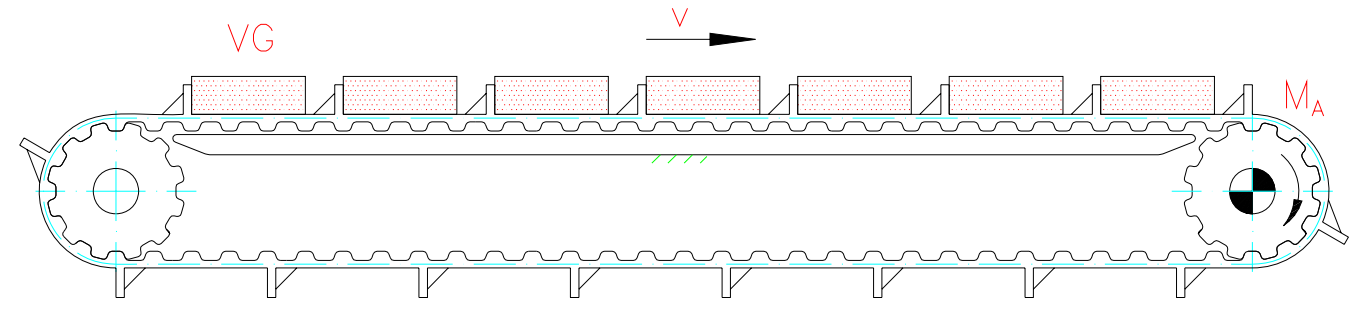

Abbildung 1: Formschlüssiger Transport mit aufgeschweißten Formelementen

Die Art und die Form des auf dem Zahnriemenrücken aufgebrachten Formelementes werden vom Gut selbst und dem Einsatzzweck bestimmt. Eine Vielzahl von verschiedenen Elementen wird von der Industrie standardmäßig angeboten.

Bei der kraftschlüssigen Variante können zwei grundlegende Arten unterschieden werden: Zum einen Zahnriemenbeschichtungen mit sehr hohem Reibwert, zum anderen mit sehr niedrigen Reibwerten.

Beschichtungen mit sehr hohem Reibwert (z. B. Silikon, PUR-Schaum, Naturkautschuk) eignen sich besonders für Schrägförderer und Abzugsbänder sowie für einfache Positionieraufgaben. Dabei wird eine relative Verschiebung des Gutes zum Zahnriemen durch den hohen Reibwert in der Kontaktzone behindert.

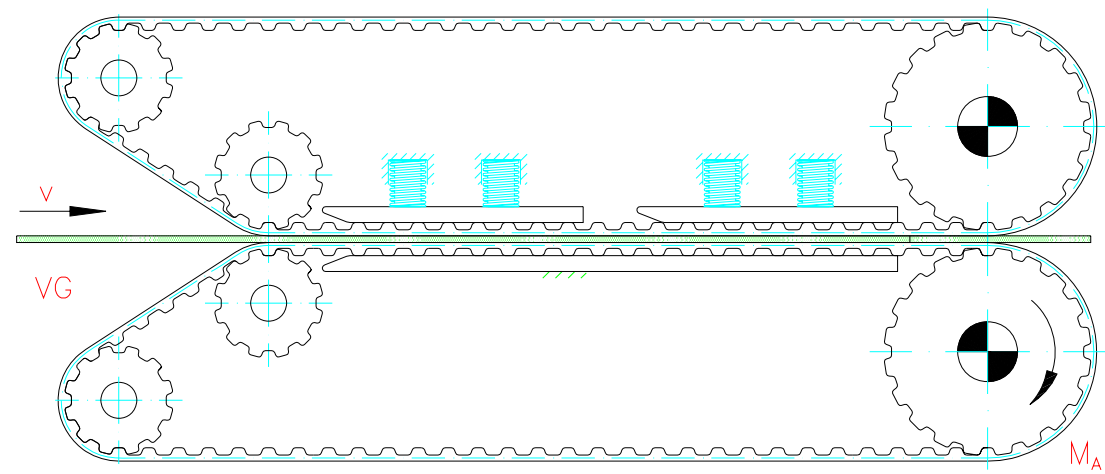

Abbildung 2: Abzugsband für biegeschlaffe Flachformkörper z. B. Folie, Textilien

Zahnriemenrückenbeschichtungen mit geringen Reibwerten bestehen meist aus Polyamidgewebe und eignen sich besonders gut für den Staubetrieb. Hierbei dient der Zahnriemen selbst als Zwischenspeicher. Bei Bedarf können die Güter freigegeben werden. Dabei muss aber sichergestellt werden, dass auch die Auflagefläche des Fördergutes für einen solchen Einsatzzweck geeignet ist, da es zu einer Relativbewegung zwischen Gut und undZahnriemen kommt.

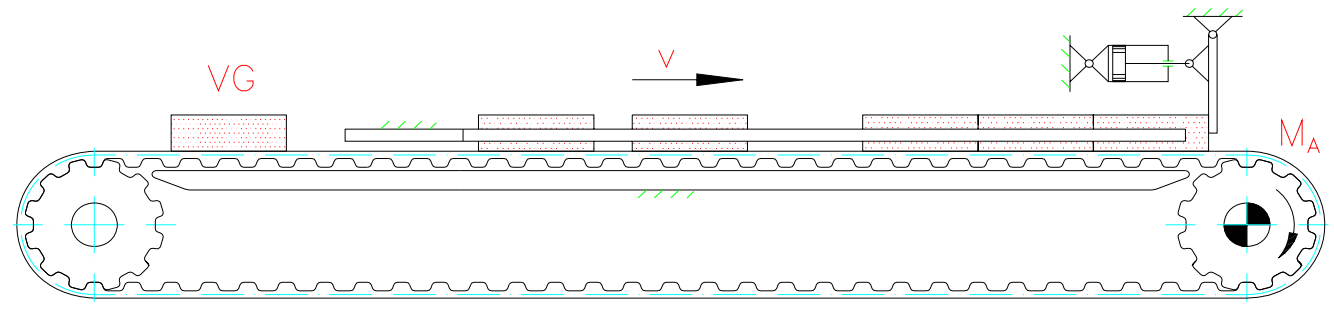

Abbildung 3: Stauförderer 
Parallelförderer können sowohl als reibschlüssige als auch als formschlüssige Variante ausgeführt werden. Ihr Vorteil liegt darin, dass größere Güter, z. B. Flachglas, Bleche usw. auf zwei oder mehreren Riemen aufliegen und durch die gleiche Geschwindigkeit der synchron angetriebenen Riemen keine Verschiebung des Gutes erfolgt. Würde der Antrieb nicht über Zahnriemen erfolgen, sondern über Flachriemen bzw. Gurte, wäre außerdem ein Zugmittel in der Breite des Fördergutes notwendig. Daraus ergibt sich zusätzlich eine wesentliche Massereduzierung der Zugmittel für den Stückguttransport in der Fördertechnik.

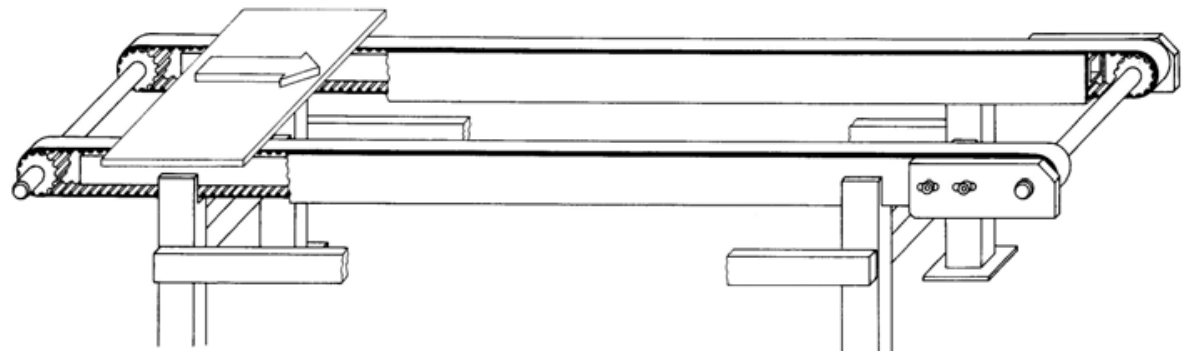

Abbildung 4: Parallelförderer für kraftschlüssigen Transport

Allen diesen Varianten ist jedoch gemein, dass der Zahnriemen auf einer Stützschiene gleitet und die Normalkraft des Transportgutes in Verbindung mit Riemengeschwindigkeit und Reibwert eine Reibleistung und damit Wärme erzeugt.

Zum gegenwärtigen Zeitpunkt fehlen exakte Angaben zu den Reibwerten für die einzelnen Gleitpaarungen. Auch ist eine Veränderung dieser Reibwerte bei Geschwindigkeits-, Temperatur-, und Belastungsänderung ungeklärt. Des Weiteren ist es auch notwendig, die Belastungsgrenzen für die Auslegung von Zahnriemenförderern zu definieren und das Verschleißverhalten zu kennen.

Die derzeit üblichen Auslegungskriterien für diese fördertechnischen Anlagen sind Zugstrangfestigkeit und Zahnfußfestigkeit. Dabei bleibt jedoch die Erwärmung des Zugmittels und der Stützschiene durch die eingebrachte Reibleistung und den sich ändernden Reibwert unbeachtet. Insbesondere bei kurzen Förderstrecken mit großen Lasten bzw. hohen Transportgeschwindigkeiten ist die Gefahr von thermischen Überlastungen gegeben, welche zu erhöhtem Verschleiß bzw. zum Totalausfall der Anlage führen kann.

Soll dieses zusätzliche Auslegungskriterium angewandt werden, sind Erkenntnisse aus den Gebieten der Tribologie und der Wärmelehre/Thermodynamik anzuwenden. Zum einen ist eine Bestimmung der entstehenden Reibleistung notwendig und zum anderen der abgeführte Wärmestrom zu ermitteln. Die sehr komplexen $\mathrm{Zu}-$ sammenhänge werden durch konstruktive und technologische Größen beschrieben, welche sich wiederum gegenseitig beeinflussen.

\section{Reibwerte in der Gleitpaarung}

In DIN ISO 7148-2 sind die Besonderheiten bei der tribologischen Prüfung von polymeren Werkstoffen beschrieben. Dabei wird explizit darauf hingewiesen, dass die Prüfanordnung möglichst der praktischen Anwendung entsprechen sollte, um die Übertragbarkeit der Prüfergebnisse zu gewährleisten.

Deshalb wurde ein Versuchsstand konzipiert, der die Kontaktverhältnisse von Zahnriemen und Stützschienen möglichst real abbildet (Abb.5). 


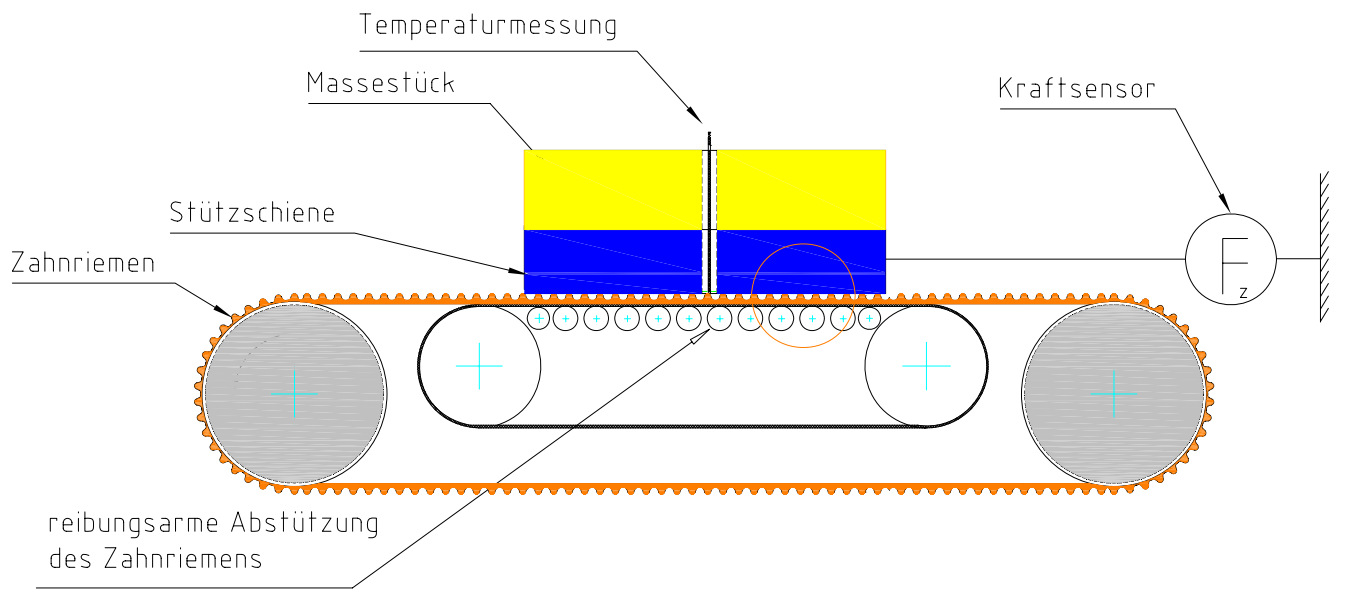

Abbildung 5: Schematischer Aufbau des Versuchsstandes

Für die Untersuchung der Zahnriemenpaarung wird der Zahnriemen mit der Zahnseite nach oben aufgespannt. Auf die nach oben zeigende Zahnseite wird eine planparallele Platte des jeweiligen Gleitschienenmaterials aufgelegt. Die Flächenpressung der Paarung lässt sich über aufgebrachte Massestücke variieren und die Reibkraft über den Kraftsensor direkt über eine Schnittstelle zur Aufzeichnung an einen Rechner weiterleiten. Zur Ermittlung der Kontakttemperaturen wurden Bohrungen in das Gleitschienenmaterial eingebracht, die unmittelbar bis an die Oberfläche der Kontaktfläche reichen und mit Thermoelementen bestückt sind. Die Abstützung des Zahnriemens erfolgt auf einem Flachriemen, der wiederum auf einer Rollenbahn abrollt. Dadurch wird ein zusätzlicher Wärmeeintrag durch eine gleitende Abstützung vermieden. Die Gleitgeschwindigkeit und Flächenpressung auf die Paarung werden in Stufen variiert. Als Versuchszahnriemen dienten PU-Riemen mit und ohne zahnseitiger Polyamidbeschichtung der Abmessung 1250 x 25 T10.

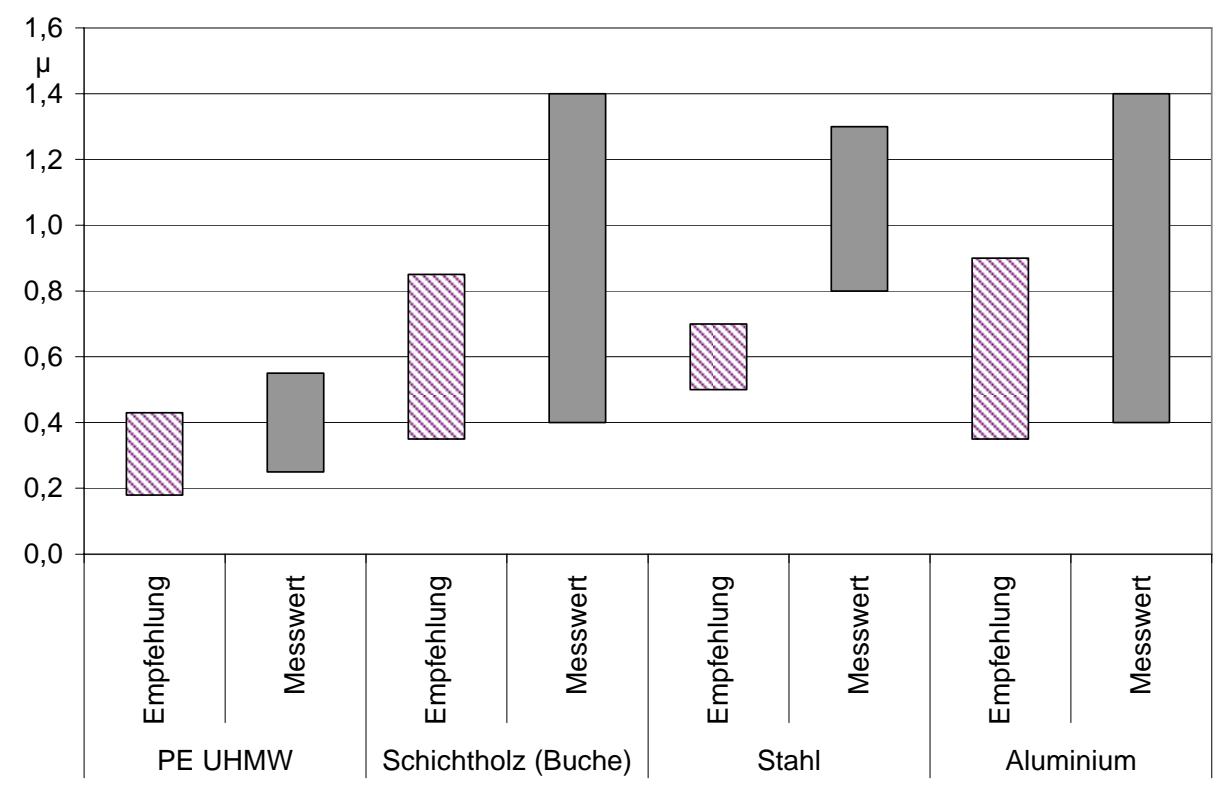

Abbildung 6: Reibwertmessungen an PU-Zahnriemen ohne Beschichtung (Kurzzeitversuche)

Die ermittelten Messwerte der Gleitreibungszahl $\mu$ für verschiedene PU-Zahnriemen - Stützschienenkombinationen sind in Abbildung 6 dargestellt. Die schraffierten Balken geben die Reibungszahlempfehlungen von Herstellern sowie aus Literaturquellen für diese Paarungen wieder. Oft wird jedoch darauf hingewiesen, dass für einen konkreten Anwendungsfall eigene Untersuchungen durchzuführen sind. Die grauen Balken geben die bei einer Versuchsdauer von bis zu 8 Stunden ermittelten Reibungszahlen wieder. Dabei wurden sowohl die 
Flächenpressungen als auch die Gleitgeschwindigkeiten variiert. Bei einigen Paarungen (Holz (Abb.7)) konnte ein sehr starker abrasiver Verschleiß am Zahnriemen festgestellt werden. Diese Werkstoffkombinationen sind nur für geringe Belastungen geeignet.

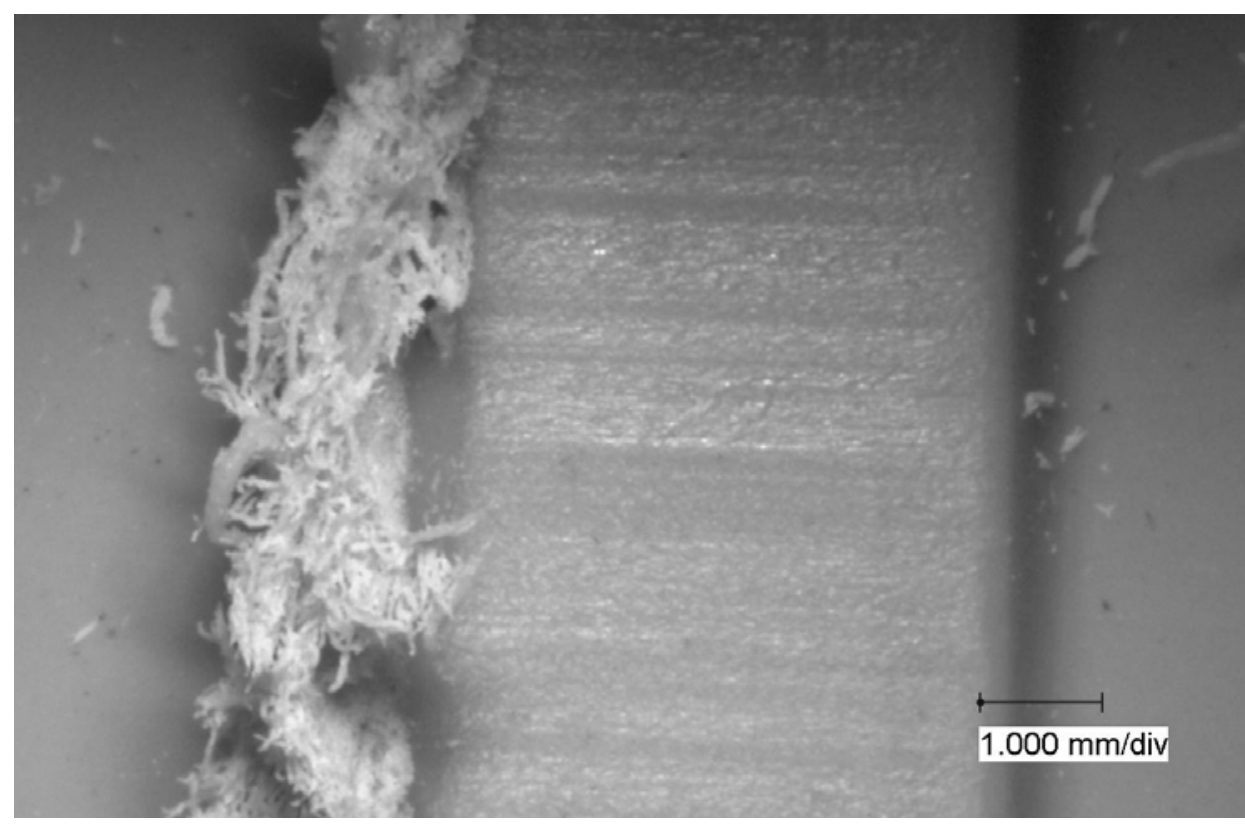

Abbildung 7: Oberfläche PU-Zahnriemen, verschlissen an Schichtholz

Die Paarungen in Verbindung mit Stahl- bzw. Aluminiumstützschienen neigen zu stick-slip- Erscheinungen verbunden mit starker Geräuschentwicklung.

Aufgrund der relativ hohen Reibungszahlen wurden keine Dauerversuche an unbeschichteten PU-Zahnriemen durchgeführt. Für die weiteren Untersuchungen wurden ausschließlich polyamidbeschichtete Zahnriemen verwendet.

In Abbildung 8 werden die Ergebnisse der Reibwertuntersuchungen an PAZ-Zahnriemen (Polyamidgewebebeschichtung auf der Zahnseite) dargestellt. Die schraffierten Balken stellen wiederum die bisherigen Empfehlungen dar, die grauen Balken die ermittelten Messwerte im Kurzzeitversuch (bis 8 Stunden) und die schwarzen Balken die Messwerte im Langzeitversuch (zwischen 7 und teilweise bis zu 100 Tagen). Hier ist die Übereinstimmung der Reibungszahlen zwischen Empfehlungen und Kurzzeitmesswerten sehr gut. Der deutliche Anstieg der Werte im weiteren Verlauf der Untersuchungen deutet daraufhin, dass der tribologische Einlauf innerhalb von 8 Stunden meist noch nicht abgeschlossen ist und dass nach fortlaufender Belastung weitere tribologische Phänomene die Kontaktverhältnisse ändern. 


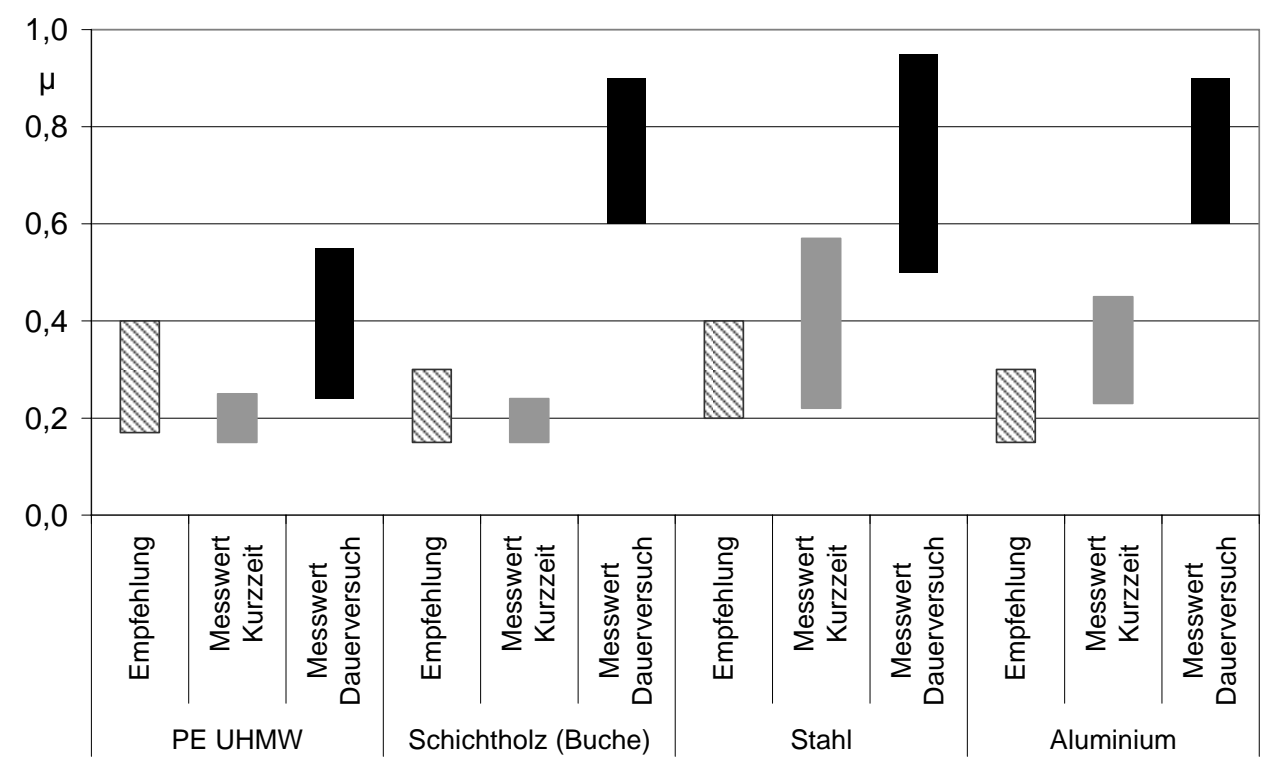

Abbildung 8: Reibungszahlen an polyamidbeschichteten PU-Zahnriemen (PAZ) in Verbindung mit verschiedenen Gleitschienen

Bei den Paarungen mit einer Stützschiene aus Stahl, Aluminium oder Schichtholz konnte eine polymere Filmbildung auf der Gleitfläche beobachtet werden. In Abbildung 9 und 10 ist die Entwicklung am Beispiel von Stahlproben zu sehen. Gemeinsam bei diesen Paarungen ist die fortschreitende Schichtbildung, verbunden mit einer Reibwerterhöhung. Der Verschleiß der Gewebeschicht am Zahnriemen setzt bei größeren Reibungszahlen ein, was zu deren weiterer Erhöhung führt Ein weiterer Einsatz führt zur vollständigen Abtragung der Gewebeschicht und damit zu einer neuen tribologischen Paarung PU-Zahnriemen - Polymerschicht.

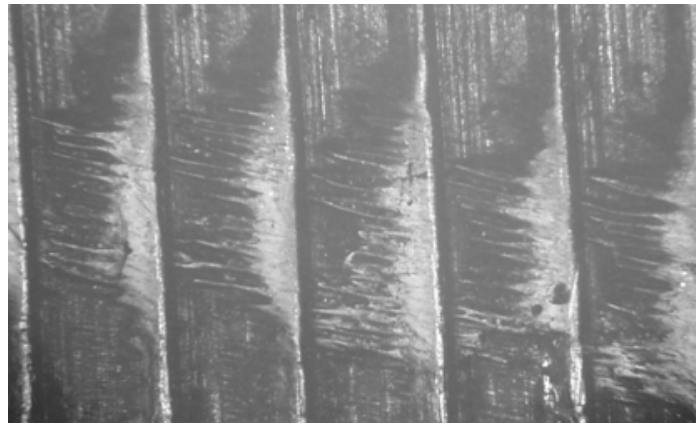

Abbildung 9: beginnende polymere Ablagerung auf Stahlprobe Rz28

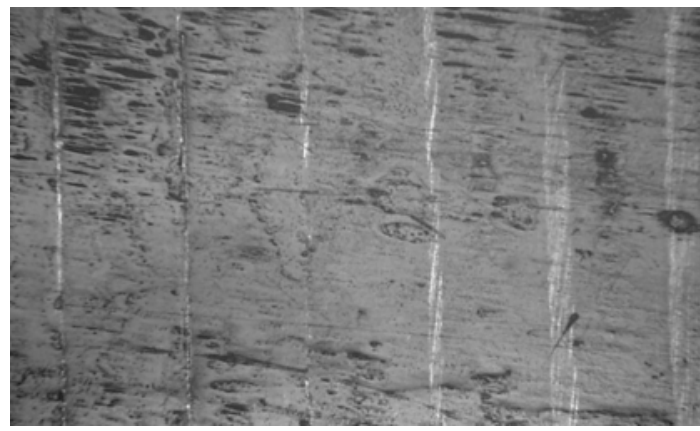

Abbildung 10: nahezu geschlossener polymerer Film auf Stahlprobe Rz28

Am Beispiel der Paarung PAZ Zahnriemen - Stahlstützschiene wird die Entwicklung der Reibungszahl über die Zeit des Gleitkontaktes in Abbildung 12 dargestellt. Dabei wurde die Oberflächenrauigkeit (Rz 6,3; Rz 28) durch 
entsprechende Bearbeitungen variiert. Der relativ starke Anstieg an der Paarung Rz 6,3 kann zum einen auf die hohe Gleitgeschwindigkeit und den damit entsprechend langen Gleitweg zurückgeführt werden, zum anderen auf den höheren adhäsiven Anteil durch die relativ glatte Oberfläche und der damit erhöhten Kontaktfläche. Abbildung 11 zeigt einen verschlissenen Zahnkopf.

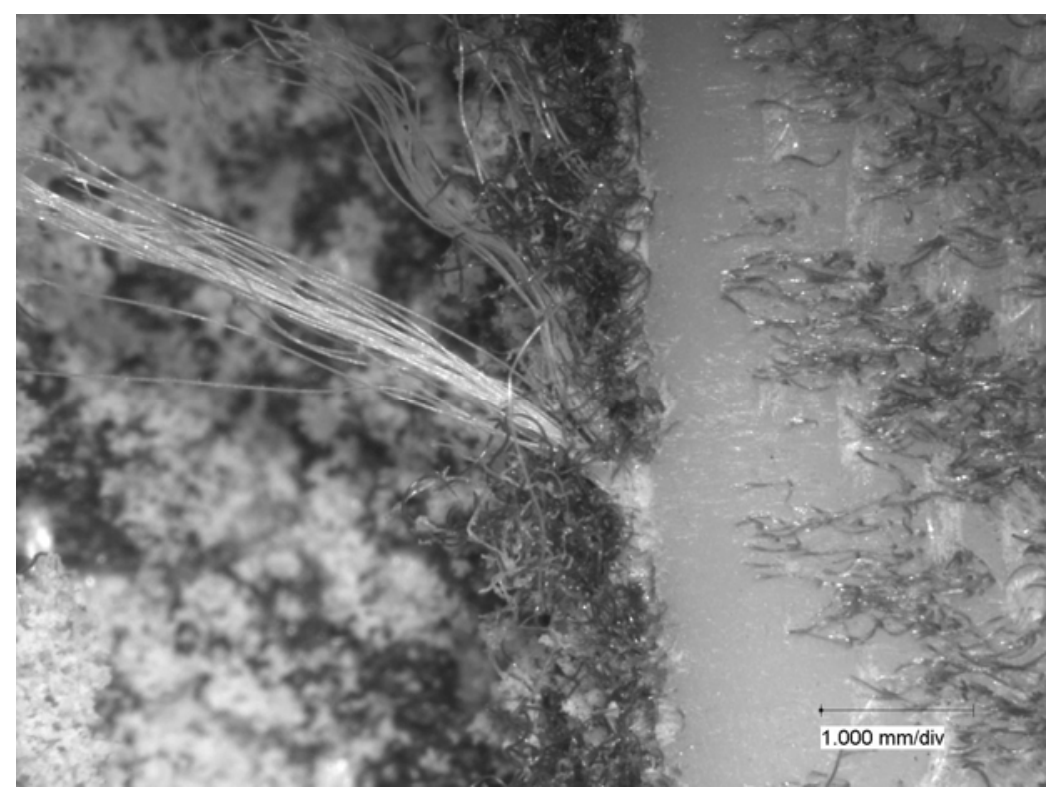

Abbildung 9: Verschlissene Zahnkopfflanke, PAZ - Stahl

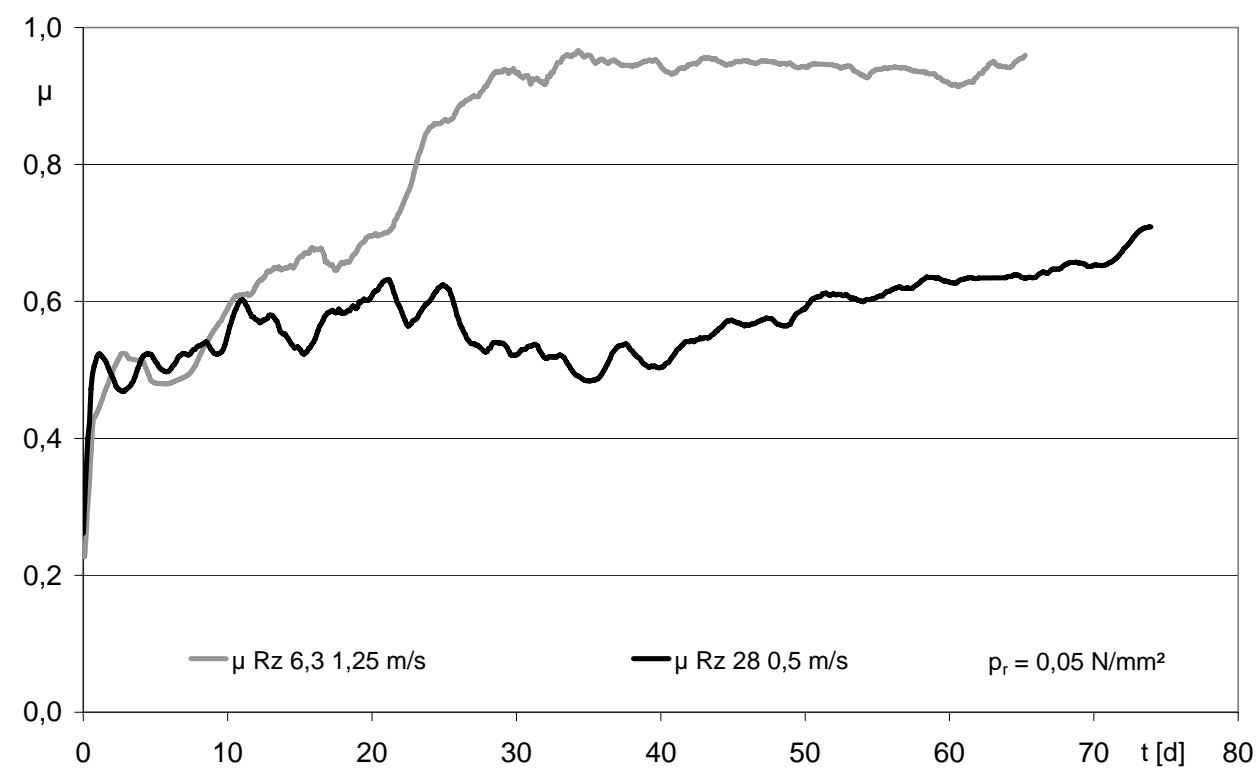

Abbildung 10: Änderung der Reibungszahl im zeitlichen Verlauf an der Paarung ZR PA - Stahl

Die Erhöhung der Reibungszahlen an der Paarung PE UHMW - polyamidbeschichteter Zahnriemen kann nicht unmittelbar auf direkte Verschleißerscheinungen zurückgeführt werden. Sowohl die Gleitfläche als auch der Zahnriemen weisen auch nach längerem Kontakt keine sichtbaren Schäden auf: Es bildet sich kein polymerer Film auf der PE- UHMW- Gleitfläche heraus. In Abbildung 11 wird die Änderung der Reibungszahl dargestellt. Es wurden Paarungen mit steigendem p•v-Wert gewählt. Mit höheren Werten für die eingebrachte Leistung pro Flächeneinheit ist ein schnellerer Anstieg der Reibungszahlen zu verzeichnen. 


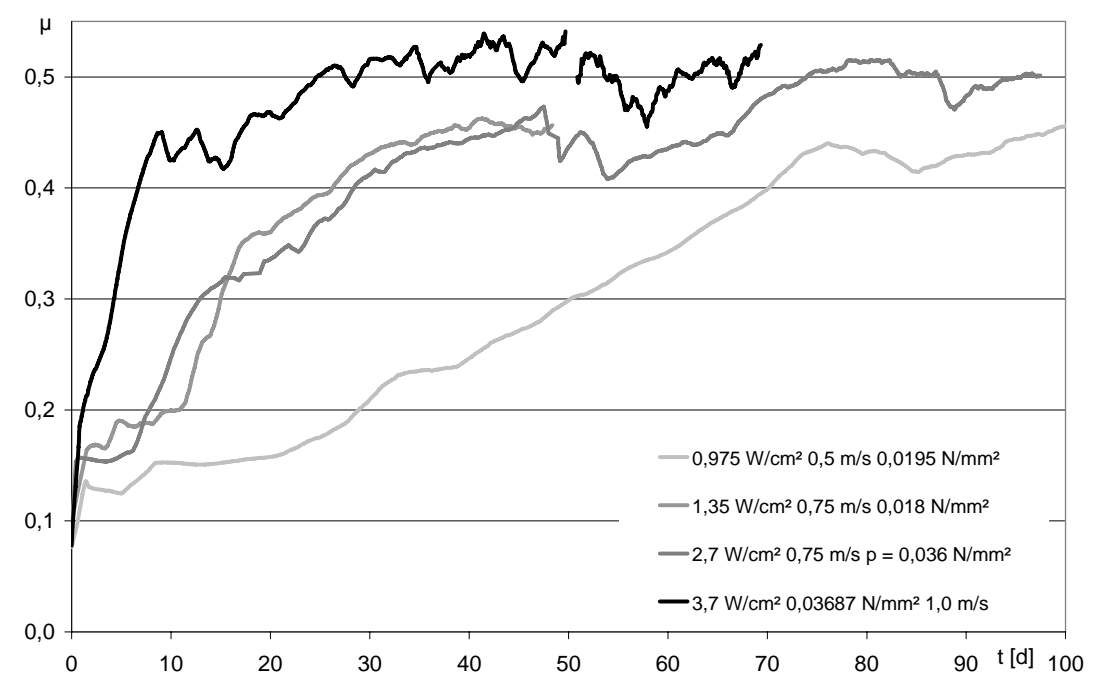

Abbildung 11: Änderung der Reibungszahl im zeitlichen Verlauf an der Paarung ZR PAZ - PE UHMW

Die Erhöhung der Reibwerte zieht nicht nur eine Steigerung der Antriebsleistung nach sich, sondern auch eine Zunahme der Reibleistung und damit einen Anstieg der Kontakttemperatur. Hat diese einen bestimmten Wert erreicht, kommt es zum Aufschmelzen der Gleitflächen und damit zum Totalausfall der Paarung (Abbildungen 14, 15, 16). Ebenfalls tritt durch die Reibwerterhöhung eine höhere Belastung des Zugstranges und der Zahnfüße im Einlauf des Zahnriemens auf. Für eine konstruktive Auslegung entsprechender Zahnriemenförderer ist dies durch entsprechende Sicherheitsfaktoren zu berücksichtigen.

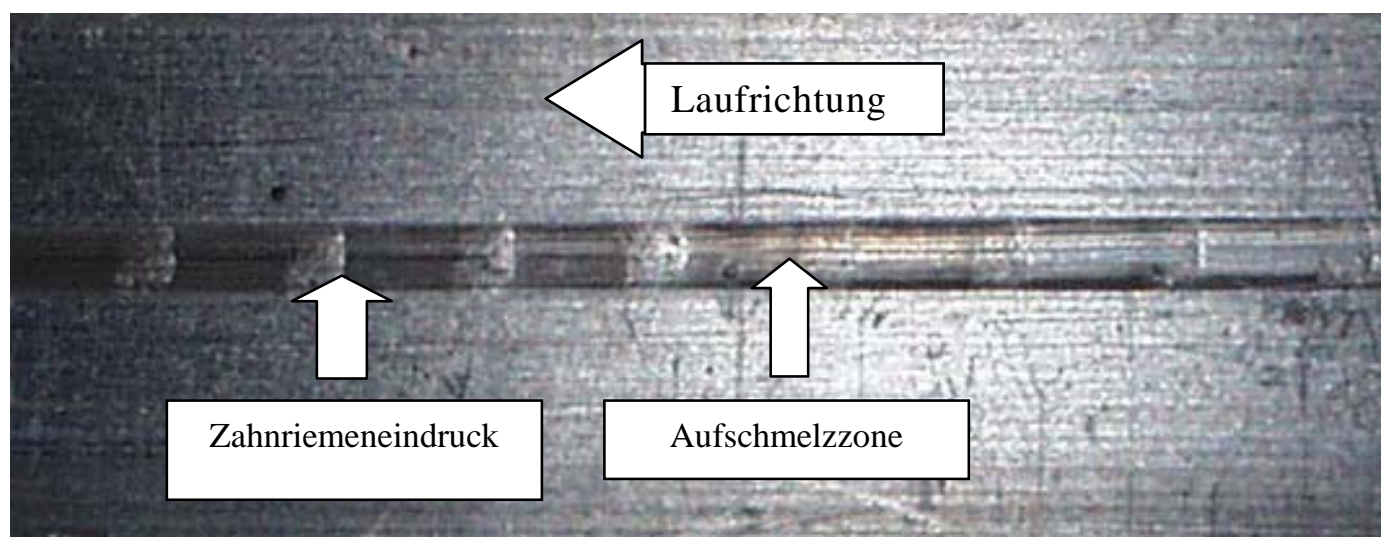

Abbildung 12: Aufgeschmolzene PE-Laufschiene, 2-fach vergrößert 


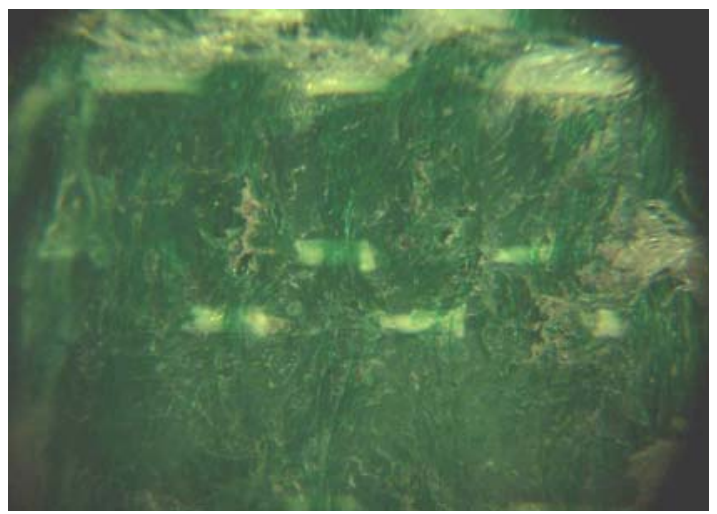

Abbildung 13: geschmolzene Faserbündel 20- fach

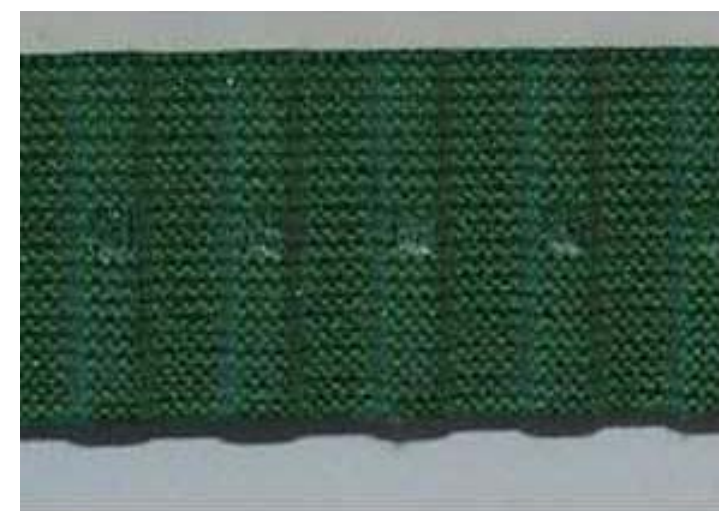

Abbildung 14: zerstörtes Gewebe in Folge thermischer Überlastung

\section{Thermische Zusammenhänge}

Die Temperaturerhöhung in der Wirkstelle zwischen Zahnriemen und Stützschiene kann im stationären Zustand in der vereinfachten Form:

$$
\Delta T=\frac{p \cdot A \cdot \mu \cdot v}{2 \cdot A \cdot\left(\frac{1}{\frac{1}{\alpha_{1}}+\frac{s_{1}}{\lambda_{1}}}+\frac{1}{\frac{1}{\alpha_{2}}+\frac{s_{2}}{\lambda_{2}}}\right)}
$$
p Flächenpressung
v Gleitgeschwindigkeit
$\mu$ Reibungszahl

A Kontaktfläche / jeweilige Oberfläche

a Wärmeübergangskoeffizient

1 Wärmeleitwert

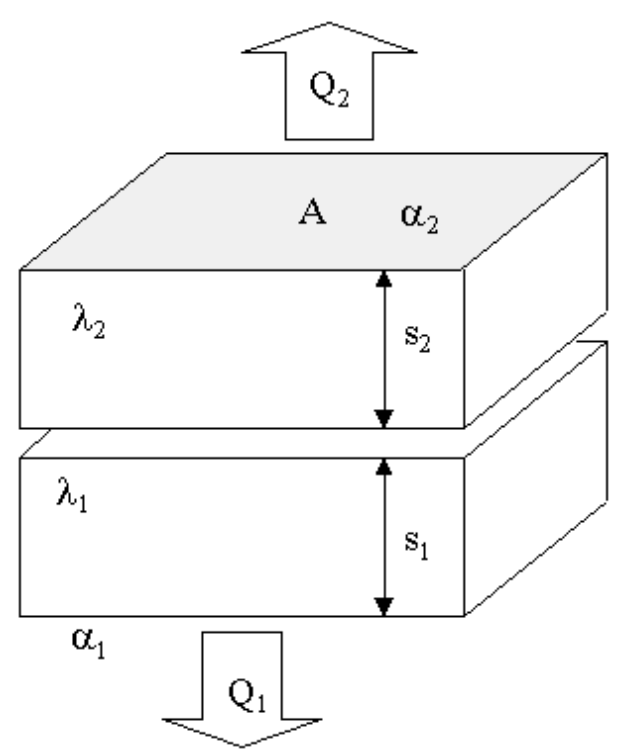

Abbildung 15: Kontaktmodell

dargestellt werden. 
Dabei werden verschiedene Vereinfachungen angenommen:

- Reibleistung wird auf die gesamte Fläche gleichmäßig verteilt,

- Wärmestrom erfolgt nur in Normalenrichtung zur Gleitfläche,

- $\quad$ konstante Reibleistung über die gesamte Zeit,

- $\quad$ keine Ableitung des Wärmestromes über Stirn- und Seitenflächen,

- $\quad$ eingeschwungener Gleichgewichtszustand der Temperaturverteilung,

- gleiche Temperatur über der jeweiligen Oberfläche,

- gleiche Strömungsverhältnisse und -bedingungen an der jeweiligen Oberfläche,

- $\quad$ konstante $\lambda$ - und $\alpha$ - Werte über der gesamten Fläche.

Der Temperaturverlauf für verschiedene Materialpaarungen ist in Abbildung 16 dargestellt. Der unterschiedliche Verlauf der Kurven kann mit den verschiedenen eingebrachten Reibleistungen durch sich unterschiedlich einstellende Reibungszahlen und durch die unterschiedlichen Wärmeleitwerte und Wärmekapazitäten der Gleitschienen erklärt werden. Ist eine stationäre Temperatur erreicht, so gilt vereinfacht die Vorstellung von Abbildung 15.

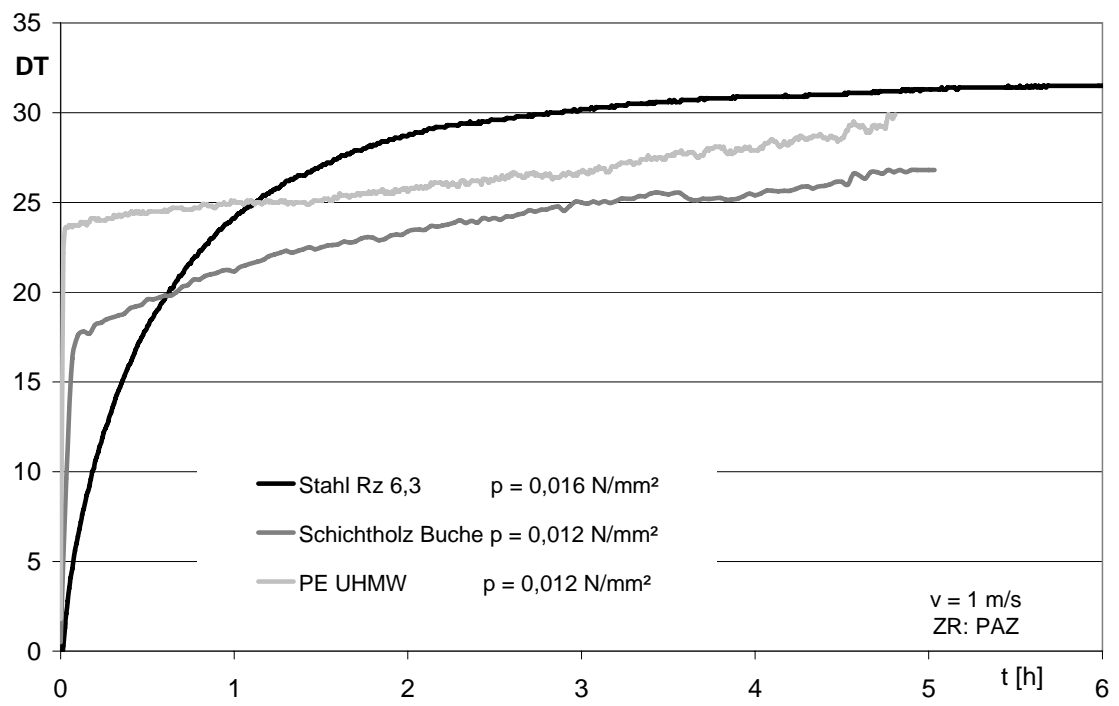

Abbildung 16: thermischer Einlauf verschiedener Stützschienenmaterialien

Die sich einstellende Gleitflächentemperatur ist im Wesentlichen von den in Abbildung 17 dargestellten Einflüssen abhängig. Da die Kontakttemperatur die Grenztemperatur (ca. $65^{\circ} \mathrm{C}$ ) nicht überschreiten darf, um eine thermische Schädigung zu vermeiden, sind die entsprechenden Einflussgrößen zweckmäßig zu wählen. Die Parameter Gleitgeschwindigkeit und Flächenpressung sind meist durch die technologischen Erfordernisse vorgegeben, die Reibungszahl stellt sich entsprechend der tribologischen Paarung ein und die Wärmeleitfähigkeit ist ein kaum zu verändernder Stoffwert. Die Einflussmaßnahmen erstrecken sich also meist auf die Schichtstärke s der Abstützung und den Wärmeübergang zur Umgebung. 


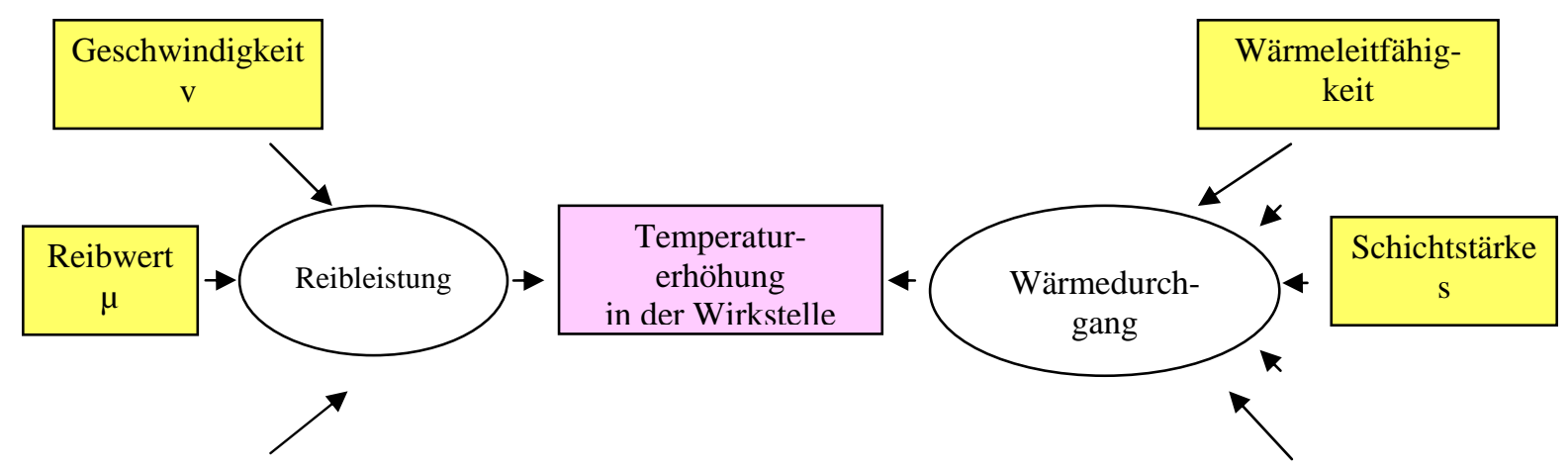

Flächenpressung $\mathrm{p}_{\mathrm{N}}$
$\alpha=\mathrm{f}(\Delta \mathrm{T}$; Oberfläche; Temperaturverteilung; Oberflächenstruktur; Turbulenzgrad; Strömungsverhältnisse;...)
Wärmeübergang

$\alpha$

Abbildung 17: Technologische und konstruktive Einflüsse auf die Gleitflächentemperatur in der Wirkstelle

\section{Zusammenfassung}

Die Kenntnis der sich nach einer entsprechenden Einlaufphase einstellenden Reibungszahlen für verschiedene Zahnriemen - Stützschienenkombinationen ist für die Anwender und Entwickler wichtig, da damit eine optimale Auslegung von Zahnriemenförderern erfolgen kann. Diese optimale Auslegung realisiert dann in der Anwendung eine ökonomische Lebensdauer bei verbesserter konstruktiver Auslegung.

Die bisher weitgehend unbeachtete Temperaturerhöhung in der Gleitschienen - Zahnriemenkombination durch die eingebrachte Reibleistung sollte zukünftig ein weiteres Auslegungskriterium darstellen. Eine erste Annäherung an dieses Problem kann durch die Form:

$$
\Delta T=\frac{p \cdot v \cdot A \cdot \mu}{2 \cdot A \cdot K}
$$

p Flächenpressung

v Gleitgeschwindigkeit

$\mu \quad$ Reibungszahl

A Kontaktfläche / jeweilige Oberfläche

K Wärmeabgabekoeffizient

DT max. zul. Temperaturerhöhung

$\mathrm{K}=\mathrm{f}(\mu, \mathrm{p}, \mathrm{v}$, Gleitschienenmaterial, Zahnriemenausführung, Maschinenkonstante...) 
gezeigt werden. Für die Ermittlung des Wärmeabgabekoeffizienten sind entsprechende Untersuchungen durchzuführen und Zusammenhänge zu ermitteln. Bestimmte Praxiseinflüsse (Umgebungstemperaturschwankungen, Verschmutzung, Stöße, Montagefehler) sind in die bisherigen Untersuchungen noch nicht eingeflossen, sollten aber nicht unbeachtet bleiben.

Durch eine vorteilhafte Auslegung der Förderanlagen kann eine höhere Zuverlässigkeit bei geringeren Wechselintervallen und niedrigeren Kosten für den Betrieb erreicht werden. 